\title{
Entwicklung einer Sensor-Überwachung für Biogasanlagen auf Basis von Prozessdaten einer Parallelanlage
}

Simone Groebel ${ }^{1,2}$, Carl Frederik Werner ${ }^{1,3}$, Marc Reimann ${ }^{1}$, Niklas Jörres ${ }^{1}$, Florian Jansen ${ }^{1}$, Katharina Kasper ${ }^{1}$, Johannes Schiffels ${ }^{1}$, Bertold Sprenger ${ }^{2}$, Marcus E.M. Baumann ${ }^{1}$, Michael J. Schöning ${ }^{1,3}$, Thorsten Selmer ${ }^{1}$,

${ }^{1}$ Institut für Nano- und Biotechnologien (INB), FH Aachen, Campus Jülich, Heinrich-MußmannStr. 1, 52428 Jülich

${ }^{2}$ Mathematisch-Naturwissenschaftliche Fakultät, Universität Rostock, Wismarsche Str. 8. 18057 Rostock

${ }^{3}$ Peter Grünberg Institut (PGI-8), Forschungszentrum Jülich GmbH, Wilhelm-Johnen-Straße, 52525 Jülich

\section{Einleitung und Motivation}

Beim Ausbau nachhaltiger, regenerativer Energieversorgung hat die Umwandlung von organischer Biomasse in Biogas ein großes Potential. Der zugrundeliegende, komplexe biologische Prozess wird noch immer unzureichend verstanden und bedarf systematischer Untersuchungen der Prozessparameter, um einen hohen Ertrag bei guter Gasqualität zu ermöglichen. Die Fragestellungen zur Entschlüsselung des Prozesses sind sowohl verfahrenstechnischer als auch mikrobiologischer Natur. Aus mikrobiologischer Sicht ist die Kenntnis der tatsächlich beteiligten prozesstragenden Mikroorganismen von erheblicher Bedeutung, aus verfahrenstechnischer Sicht die Kenntnis der physikalischen und chemischen Faktoren, welche die mikrobiologischen Prozesse und kontrollieren. Im Zusammenspiel aller dieser Parameter wird die Biogasbildung befördert oder behindert, bis zum Abbruch des Prozesses.

Eine mögliche Kontrollmethode ist die Messung der metabolischen Aktivität prozesstragender Organismen. Diese soll, beruhend auf fundierten Prozessdaten, gewonnen durch eine Parallelanlage, mit einem lichtadressierbaren potentiometrischen Sensor-System (LAPS) realisiert werden. Dieser Sensor ist in der Lage, pH-Wert-änderungen zu detektieren, die durch den Stoffwechsel der auf dem Chip immobilisierten Organismen hervorgerufen werden (siehe Bild 1), um eine Online-Überwachung von Biogasanlagen zu ermöglichen.

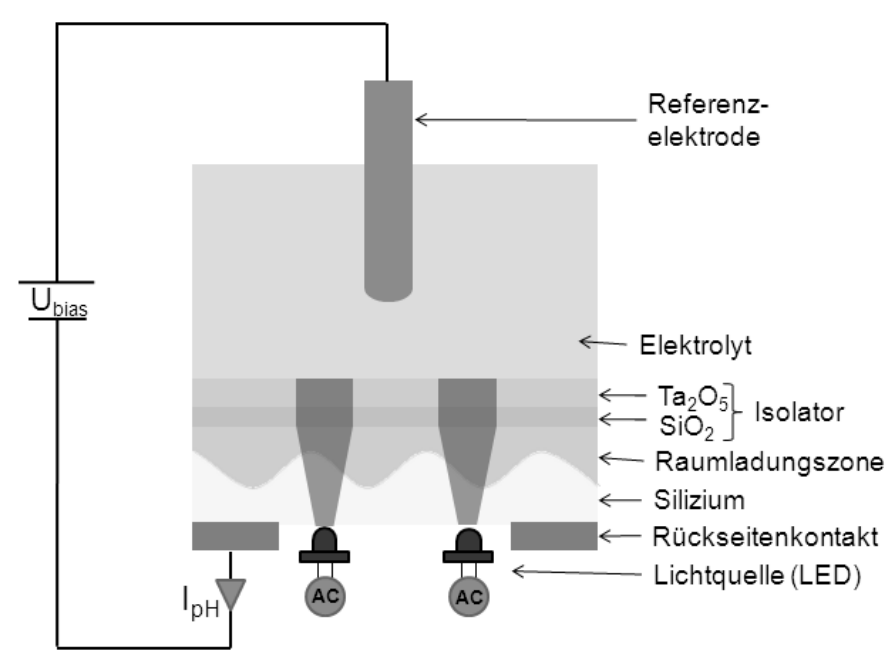

Bild 1 Schematischer Messaufbau des LAPS, der einseitig mit Organismen immobilisiert ist. Unterhalb des Sensors befinden sich die Lichtquellen (LED's) und die Messspots. Oberhalb, in die Lösung eingetaucht, befindet sich eine Referenzelektrode. 


\section{Material und Methoden}

Zur Untersuchung der Prozessparameter wurde ein vollautomatisiertes Fermentersystem, bestehend aus sechs identischen Reaktoren, im Labormaßstab aufgebaut (vgl. Bild 2). Jeder einzelne Fermenter (1 L Volumen) wurde mit individuell steuerbarer Heizung, Durchmischung und kontinuierlicher Fütterung ausgestattet. Regelung und Steuerung der Anlage erfolgt LabVIEW-basiert. Die entscheidenden Prozessparameter wie Temperatur, $\mathrm{pH}-$ Wert, Redoxpotential und die Gasqualität und -quantität werden fortlaufend erfasst und überwacht.
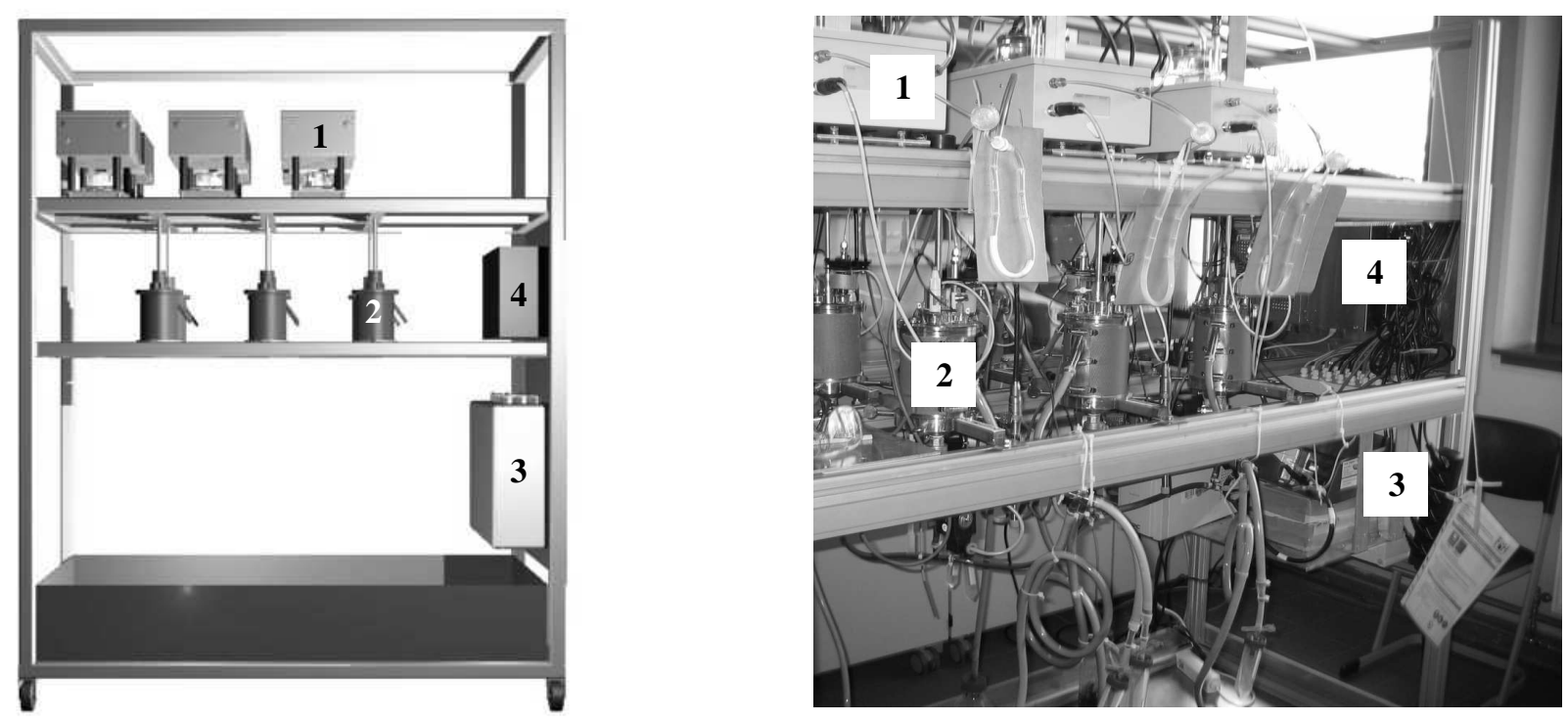

Bild 2 Schematische Darstellung und realer Aufbau der Parallelanlage zur systematischen Untersuchung des Biogasprozesses. Mittig (2) befinden sich sechs identische 1 L Fermenter, die jeweils mit einer darüber liegenden Gasanalytik (1) verbunden sind. Unterhalb der Fermenter befindet sich die automatisierte Fütterung, bestehend aus einer Pumpe und einem Vorratsgefäß. Die Regelungs- und Auswertesoftware (3) ermöglicht über einen Panel-PC (4) die Einstellung der Prozessparameter sowie die Auslesung der gewonnenen Daten.

Im Rahmen der Untersuchungen wurde zunächst überprüft, ob identisch betriebene Reaktoren auch vergleichbare und reproduzierbare Ergebnisse liefern. Dies war die Grundvoraussetzung, für die Interpretierbarkeit der Ergebnisse der weitergehenden Versuche. In ersten Experimenten wurden die optimalen Parameter des Systems getestet. Zu den Parametern, die von höchstem Interesse für die Bildung von Methan sind, gehören die Temperatur, die Raum-Zeit-Belastung, und der Einsatz von Co-Substraten. In weiteren Versuchen wurde der Prozess hinsichtlich seiner Stabilität untersucht. Dazu wurden gezielt organische Fettsäuren, die wichtigsten Intermediärprodukte, zugegeben.

Diese Daten aus den Prallelansätzen dienen als Basis des zu entwickelnden Sensors. Zu Beginn erfolgte dafür ein „Proof of principle“. Dazu wurden eine Differenzanordnung auf einem Chip realisiert und Messungen mit dem Testorganismus E. coli durchgeführt.

\section{Ergebnisse und Diskussion}

\subsection{Vorversuche der Anlage}

In Vorversuchen hat sich gezeigt, dass sich die Parallel-Reaktoren nahezu identisch verhalten, wenn diese unter den gleichen Bedingungen betrieben werden. Aufgrund dessen können entscheidende Prozessparameter einzeln untersucht werden, um deren Auswirkungen auf den Biogas-Prozess im Labormaßstab zu studieren. 
Ergänzend wurden die Verfahrensparameter untersucht. Als optimal und damit als Grundeinstellung aller weiteren Versuche wurde eine Temperatur von $37{ }^{\circ} \mathrm{C}$ ermittelt. Die Durchmischung erfolgte kontinuierlich mit einer Rührgeschwindigkeit von $44 \mathrm{rpm}$. Die Fütterung wurde auf ein Intervall programmiert, so dass sechs Mal täglich eine Gesamtzufütterung von $10 \mathrm{~kg}$ Organik pro $\mathrm{m}^{3}$ erfolgte. Zusätzlich konnten gezielt Prozessstörungen hervorgerufen und analysiert werden.

\subsection{Untersuchung gezielter Störungen}

Gezielt wurde der Prozess zur genaueren Analyse gestört. Dazu wurden, mit Ausnahme eines Kontrollfermenters (Fermenter A), den Fermentern organische Fettsäuren zudosiert. Diese, auch im natürlichen Betrieb vorliegenden Säuren, waren Acetat (Fermenter B), Propionat (Fermenter C), Butyrat (Fermenter D), Isobutyrat (Fermenter E) und Phenylessigsäure (Fermenter F). Der gesamte Versuch staffelte sich in drei Teile. Im ersten Teil wurden alle sechs Fermenter identisch angefahren und bei konstanten Bedingungen betrieben (Tag 0-6). Anschließend wurden an Versuchstag sechs die Konzentrationen auf $150 \mathrm{mmol} / \mathrm{L}$ Acetat in Fermenter B angehoben. In den restlichen Fermentern wurden die Säuren auf eine Endkonzentration von $50 \mathrm{mmol} / \mathrm{L}$ eingestellt. Am 14. Versuchstag wurden den einzelnen Fermentern erneut organische Säuren zugeführt, diesmal in doppelter Konzentration zum vorherigen Versuch. Im Folgenden (Bild 3) sind beispielhaft die entstandenen Gasmengen sowie die Anteile an Methan und Kohlenstoffdioxid des Kontrollfermenters (Fermenter A) und der Zugaben von Acetat, Propionat und Butyrat dargestellt.
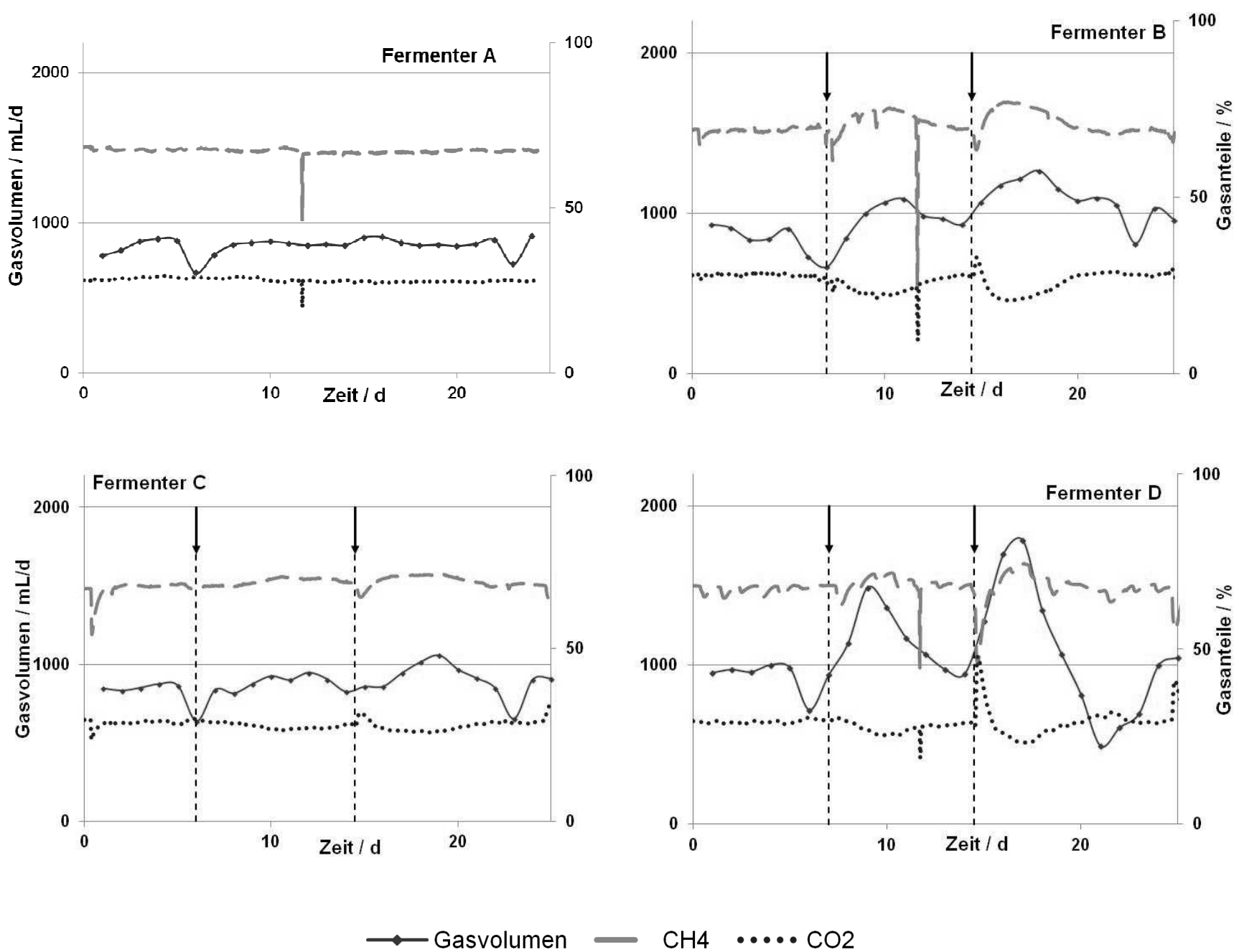

Bild 3 Entwicklungen der Gasbildungsraten und die Veränderungen der Gasqualitäten durch die Zugabe von organischen Säuren. Fermenter A, der ohne Zugabe als Kontrolle fungierte, zeigt konstante Gasqualitäten und leicht schwankende Gasmengen. Fermenter B, dem Acetat zugesetzt worden war, weist daraufhin steigende Gasmengen und Qualitäten auf. Die Zugaben von Propionat in Fermenter C zeigen kaum einen Einfluss. Fermenter D und die Zugaben von Butyrat erreichen maximale Gasausbeuten. 
Der Verlauf von Fermenter A, dem Kontrollfermenter, weist konstante Verläufe in Gasqualität und quantität. Somit ist eine Vergleichbarkeit möglich. Die Zugabe von Acetat bewirkt einen kurzzeitigen Anstieg von Gasqualität und -quantität. Die Anstiege fallen bei $300 \mathrm{mmol} / \mathrm{L}$ stärker aus als bei $150 \mathrm{mmol} / \mathrm{L}$. Die Zugabe von Propionat zeigt keine großen Einflüsse auf die Gasmenge und die Methan- und Kohlendioxidkonzentration, Butyrat hingegen schon. Besonders auffällig sind die Anstiege in der Gasmenge.

\subsection{Proof of principle mit LAPS}

Die Untersuchungen mit dem Modellorganismus E. coli zeigen zum einen, dass eine Erfassung der Stoffwechselprodukte mit LAPS möglich ist. Zum anderen verdeutlichen sie die Bedeutung des Unterschieds, ob die Organismen frei in Lösung oder in einem Acrylamidgel auf dem Chip immobilisiert vorliegen (s. Werner et al., [1]).

\section{Ausblick}

Der erfolgreiche Ausbau und Betrieb der Parallelanlage ermöglicht die gezielte Untersuchung weiterer Fragestellungen. Neben dem Test weiterer Störszenarien können auch alternative Substrate gezielt getestet werden.

Die Entwicklung des Sensors soll nach dem „Proof of principle“ auf die speziellen Anforderungen im Biogasprozess angepasst werden. In diesem Rahmen soll der Sensor dafür acetatverwertende Mikroben auf seiner Oberfläche tragen. Erste Messungen haben bereits gezeigt, dass der Modellorganismus Methanosaeta concilii sehr empfindlich auf Änderungen der Acetatkonzentration reagiert und die damit verbundene Änderung seiner metabolischen Aktivität durch die pH-empfindliche Oberfläche eines LAPS-Chips ausgelesen werden kann. Im Weiteren soll auf diesen Basisdaten aufbauend, ein ähnlicher LAPS zur OnlineÜberwachung in den parallelen Reaktoren implementiert werden. Dieser könnte die Offline-Analytik ergänzen bzw. ersetzen und in Echtzeit die biologisch relevante Prozesssituation widerspiegeln, lange bevor sich physikalisch-chemische Parameter ändern.

\section{Danksagung}

Die Autoren danken dem BMELV und der Fachagentur Nachwachsende Rohstoffe e.V. für die finanzielle Förderung des Forschungsvorhabens (,Bio-LAPS“).

\section{Literatur}

[1] C.F. Werner, C. Krumbe, K. Schumacher, S. Groebel, H. Spelthahn, M. Stellberg, T. Wagner, T. Yoshinobu, T. Selmer, M. Keusgen, M.E.M. Baumann, M.J. Schöning, Phys. Status Solidi A 208(6), 1340$1344(2011)$ 